\title{
NEW FOKKER-PLANCK DERIVATION OF HEAVY GAS MODELS FOR NEUTRON THERMALIZATION
}

\author{
EDWARD W. LARSEN and M.M.R. WILLIAMS \\ Department of Nuclear Engineering \\ The University of Michigan \\ Ann Arbor, MI 48109
}

(Received 1 February 1989; in revised form 14 October 1989)

\begin{abstract}
This paper is concerned with the derivation of new generalized heavy gas models for the infinite medium neutron energy spectrum equation. Our approach is general and can be used to derive improved Fokker-Planck approximations for other types of kinetic equations. In this paper we obtain two distinct heavy gas models, together with estimates for the corresponding errors. The models are shown in a special case to reduce to modified heavy gas models proposed earlier by Corngold (1962). The error estimates show that both of the new models should be more accurate than Corngold's modified heavy gas model, and that the first of the two new models should generally be more accurate than the second.
\end{abstract}

\section{INTRODUCTION AND HISTORICAL REVIEW}

It has been many years since the heavy gas model of neutron thermalization was developed by Wilkins (1944). The purpose of Wilkins' work was to reduce an integral equation, with a complicated kernel, to a relatively simple second order differential ("heavy gas" or "Fokker-Planck") equation which would be amenable to analytical or rapid numerical solution. The purpose of this paper is to take a fresh look at this subject and show theoretically that many of the results which have been obtained since Wilkins' work can be generalized to more accurate models. Our method is very general and can be applied to Fokker-Planck approximations for other kinetic equations. However, before presenting our analysis, it will be useful to review some of the earlier results.

Apart from improved methods of derivation (Hurwitz, Nelkin and Habetler, 1956) the Wilkins heavy gas model remained unchanged for some years. The model does, however, suffer from a severe physical limitation, namely it is restricted to the description of neutron thermalization in moderators where chemical binding is unimportant, as well as to situations where $A \gg 1$. Some attempts have been made to extend its use, artificially, to describe binding effects by introducing an effective mass. Because binding generally has the effect of reducing the energy transfer between neutron and nucleus, the association of an effective nuclear mass, larger than the true mass, has the desired effect. Rigorous methods for defining the effective mass were developed by Sachs and Teller (1941). Such methods are useful for purely thermal problems, but they have the disadvantage of not leading to the correct slowing down spectrum when the neutron energy is very much greater than $k T$ : this is because in the slowing down region the mass must be the true mass and not the effective one. Some attempts to correct for this problem by the introduction of an energy dependent effective mass were not very successful because the resulting scattering kernel no longer satisfied detailed balance.

The difficulties mentioned above led, in the 1960 's, to a considerable effort to extend the heavy gas model in a consistent fashion. To understand how this development 
took place, it is useful to examine the structure of the classical heavy gas model as developed by Wilkins and in another manner by Hurwitz et al (1956). The thermalization operator

$$
L \phi(E)=\int_{0}^{\infty}\left[\Sigma_{S}\left(E^{\prime} \rightarrow E\right) \phi\left(E^{\prime}\right)-\Sigma_{S}\left(E \rightarrow E^{\prime}\right) \phi(E)\right] d E^{\prime}
$$

is represented in the Wilkins approximation by

$$
L \phi(E) \approx k T E \frac{d^{2} \phi}{d E^{2}}+E \frac{d \phi}{d E}+\phi
$$

This may also be written as

$$
L \phi \approx k T \frac{d}{d E}\left[E M(E) \frac{d}{d E}\left(\frac{\phi(E)}{M(E)}\right)\right] \quad,
$$

where the Maxwellian distribution $M(E)$ is

$$
M(E)=\frac{E}{(k T)^{2}} e^{-E / k T}
$$

It is a requirement, from the detailed balance relation

$$
\Sigma_{S}\left(E^{\prime} \rightarrow E\right) M\left(E^{\prime}\right)=\Sigma_{S}\left(E \rightarrow E^{\prime}\right) M(E) \quad,
$$

that $L M(E)=0$, and we see that Eq. (1.3) satisfies this condition. We also note that an equally valid form for $\mathrm{L}$, as far as detailed balance is concerned, is

$$
L \phi \approx k T \frac{d}{d E}\left[f(E) E M(E) \frac{d}{d E}\left(\frac{\phi(E)}{M(E)}\right)\right] .
$$

In this equation, $f(E)$ is an arbitrary function that can be chosen so that some integral property of $L$ is preserved, or so that $\phi$ satisfies an exact solution of some specific thermalization problem. This approach was followed by Schaefer and Allsop (1962), who obtained asymptotic representations of $f(E)$ for $E>k T$ by ensuring a fit with Corngold's asymptotic analysis of the slowing down problem (Corngold, 1960). Later work by Pitcher (1963), who used an experimentally determined thermal spectrum to unfold $f(E)$, should also be mentioned.

This type of approach was useful because it gave some indication of the degree of deviation of the heavy gas model from the exact results. Indeed, $f(E)$ was seen to vary significantly with energy in the region $E<k T$. Even so, these methods are limited in the sense that $f(E)$ is problem dependent and is therefore only valid in a restricted sense.

A more rigorous and consistent approach to the approximate representation of the integral thermalization operator $L$ by a second order differential operator was presented by Corngold (1962). Corngold noted that $L$ satisfies the conditions

$$
\int_{0}^{\infty} L \phi(E) d E=0
$$




$$
L M(E)=0 \quad,
$$

where $\phi(E)$ is any function. If the true scattering operator $L$ is to be replaced by a secondorder differential operator $R$,

$$
R \phi(E)=\frac{d^{2}}{d E^{2}} p(E) \phi(E)-\frac{d}{d E} r(E) \phi(E)+s(E) \phi(E) \quad,
$$

then the condition (1.6) requires $s(E)=0$. Thus,

$$
R \phi(E)=\frac{d}{d E}\left(\frac{d}{d E} p(E) \phi(E)-r(E) \phi(E)\right),
$$

and now condition (1.7) is satisfied if

$$
\frac{d}{d E} p(E) M(E)=r(E) M(E)
$$

To determine $p(E)$ and $r(E)$, Corngold requires that the additional condition

$$
\int_{0}^{\infty} E L \phi(E) d E=\int_{0}^{\infty} E R \phi(E) d E
$$

be satisfied for every $\phi(E)$. Using Eqs. (1.1) and (1.8), Eq. (1.10) reduces to

where

$$
\int_{0}^{\infty} A_{1}(E) \phi(E) d E=\int_{0}^{\infty} r(E) \phi(E) d E \quad,
$$

$$
A_{n}(E)=\int_{0}^{\infty} \Sigma\left(E \rightarrow E^{\prime}\right)\left(E^{\prime}-E\right)^{n} d E^{\prime} \quad,
$$

and hence

$$
r(E)=A_{1}(E)
$$

If one imposes the additional constraint

$$
\int_{0}^{\infty} E^{2} L \phi(E) d E=\int_{0}^{\infty} E^{2} R \phi(E) d E \quad,
$$

then one obtains

$$
p(E)=\frac{1}{2} A_{2}(E) \quad .
$$

However, the two results (1.12) and (1.14) are not both consistent with Eq. (1.9). Corngold recommends dropping Eq. (1.14), using Eq. (1.12) to define $r(E)$, and then using Eq. (1.9) to determine $p(E)$. This yields 


$$
p(E) M(E)=\int_{0}^{E} A_{1}\left(E^{\prime}\right) M\left(E^{\prime}\right) d E^{\prime}
$$

Introducing Eq. (1.11) into this result and using the fact that, by detailed balance,

$$
\int_{E^{\prime}=0}^{E} \int_{E^{\prime \prime}=0}^{E}\left(E^{\prime \prime}-E^{\prime}\right) M\left(E^{\prime}\right) \Sigma_{S}\left(E^{\prime} \rightarrow E^{\prime \prime}\right) d E^{\prime \prime} d E^{\prime}=0,
$$

one obtains

$$
p(E) M(E)=\int_{E^{\prime}=0}^{E} \int_{E^{\prime \prime}=E}^{\infty}\left(E^{\prime \prime}-E^{\prime}\right) M\left(E^{\prime}\right) \Sigma_{S}\left(E^{\prime} \rightarrow E^{\prime \prime}\right) d E^{\prime \prime} d E^{\prime} .
$$

Eqs. (1.8) and (1.9) now combine to give

$$
R \phi(E)=\frac{d}{d E} p(E) M(E) \frac{d}{d E} \frac{\phi(E)}{M(E)},
$$

where Corngold's recommended "diffusion coefficient" $p(E) M(E)$ is given by Eq. (1.15). Corngold's alternative (not recommended) diffusion coefficient, derived using Eq. (1.14) rather than Eq. (1.12), is given by

$$
\begin{aligned}
p(E) M(E) & =\frac{1}{2} M(E) \int_{0}^{\infty} \Sigma_{S}\left(E \rightarrow E^{\prime}\right)\left(E^{\prime}-E\right)^{2} d E^{\prime} \\
& =\frac{1}{2} \int_{0}^{\infty}\left(E^{\prime}-E\right)^{2} M\left(E^{\prime}\right) \Sigma_{S}\left(E^{\prime} \rightarrow E\right) d E^{\prime} .
\end{aligned}
$$

In this paper, we derive two new generalized heavy gas models for the neutron spectrum equation

$$
\Sigma_{A}(E) \phi(E)=L \phi(E)+Q(E) \quad,
$$

where the scattering operator $\mathrm{L}$ is defined by Eq. (1.1). Our main assumption is that the differential scattering cross section is sharply peaked near $E^{\prime}=E$, and hence

$$
\int_{0}^{\infty} \Sigma_{S}\left(E^{\prime} \rightarrow E\right)\left|E^{\prime}-E\right|^{n+1} d E^{\prime} \ll \int_{0}^{\infty} \Sigma_{S}\left(E^{\prime} \rightarrow E\right)\left|E^{\prime}-E\right|^{n} d E^{\prime},
$$

for $n=0,1,2$. This assumption allows us to introduce an ordering of and justify the deletion of certain terms. We do not require integral conditions, such as Eq. (1.10) or Eq. (1.13), to hold. Nevertheless, our results generalize much of the earlier work cited above.

In particular, we require knowledge of a function $\Phi(E)$ which reasonably well approximates the solution $\phi(E)$ of Eq. (1.18), and we obtain a result of the form (1.16) in which $p(E)$ depends on $\Phi(E)$. Thus, our solution depends on the particular problem to be solved, as in the cited work of Schaefer and Allsop (1962), and Potter (1960). However, if we take $\Phi(E)=M(E)$, then we show that our results identically reduce to those of Corngold (1962). This shows that if $\phi(E) \approx M(E)$ for all $E$, then Corngold's hypothesized condition (1.10) in fact follows from our analysis.

Our analysis also provides an estimate of the error involved in the generalized heavy gas approximation. This estimate shows that our new methods should be more accurate than the conventional heavy gas models, at least for certain types of problems, and for large 
energies. However, we emphasize that the work presented here is analytic in nature and has not yet been tested computationally.

It might be argued that the construction of Fokker-Planck approximations to integral operators, as is done in this paper, is unnecessary because of modern computing power. However, there are advantages to be gained by this construction. First, it highlights the physical parameters to which the problem is most sensitive and therefore gives guidance about where to concentrate numerical effort. Second, there are many problems where, after the inclusion of space and time variables, the computing effort required becomes formidable and expensive. This is especially true of other fields of transport theory involving gamma rays, electrons, or photons. For example, the Compton Scattering of photons is commonly approximated by a differential Fokker-Planck operator in computational algorithms (Pomraning, 1973, Cooper, 1971, Larsen et. al., 1985). The analysis in this paper is also applicable to this problem. Thus, the derivation of improved differential or Fokker-Planck approximations is worthwhile and will remain so, regardless of advances in computational hardware.

We also note that the reduction of an integral equation to a differential one has its roots in the theory of Markov processes. If the transition probabilities from one state to another have $n$-th moments that are $O\left(\varepsilon^{n}\right)$, where $\varepsilon$ is some small quantity, say $\Delta t$ (the transition time), then the Master equation can be reduced to a second order differential equation. Examples of such a procedure can be found in chemical reactions, where the second order differential equation is actually a three point difference equation, and also in the Rayleigh and Lorentz gases (the neutron gas is a Lorentz gas). (Oppenheim et. al., 1977).

A summary of the remainder of this paper follows. In Sec. II we derive method A, a generalized heavy gas model of the form (1.16) with a diffusion coefficient which generalizes Eq. (1.15). In Sec. III we derive method B, a generalized heavy gas model of the form (1.16) with a diffusion coefficient which generalizes Eq. (1.17). In Sec. IV we compare the two methods and argue that method A should be generally superior to method B. (Corngold argued the same thing for his two models, to which methods A and B reduce.) We conclude with a brief discussion in Sec. V.

\section{DERIVATION OF METHOD A}

In what follows, we let $\phi(E)$ denote the solution of the neutron spectrum equation (1.18). We also require there to exist a function $\Phi(E)$ such that the functions

$$
\psi(E) \equiv \frac{\phi(E)}{\Phi(E)}
$$

and

$$
\zeta(E) \equiv \frac{M(E)}{\Phi(E)}
$$

are bounded and have bounded first and second derivatives for all $0<E<\infty$. The functions $\psi$ and $\zeta$ can tend to zero, but they cannot become infinite. For example, if $\phi(E)$ is smooth and behaves like a Maxwellian for thermal energies and like $E^{-1}$ for $E \gg k T$, then $\Phi(E)$ can be any smooth nonnegative function satisfying 


$$
\Phi(E) \approx\left\{\begin{array}{ll}
M(E) & E \approx k T \\
\beta / E & E \gg>T
\end{array},\right.
$$

where $\beta$ is a suitable constant. For another example, if $\phi(E)$ behaves like a Maxwellian for all energies of interest, then one can simply take $\Phi(E)=M(E)$.

Let us now define

$$
q(E)=\int_{E^{\prime}=E}^{\infty} \int_{E^{\prime \prime}=0}^{E}\left[\Sigma_{S}\left(E^{\prime} \rightarrow E^{\prime \prime}\right) \phi\left(E^{\prime}\right)-\Sigma_{S}\left(E^{\prime \prime} \rightarrow E^{\prime}\right) \phi\left(E^{\prime \prime}\right)\right] d E^{\prime \prime} d E^{\prime},
$$

and then one can easily show that

$$
\frac{d}{d E} q(E)=L \phi(E)
$$

Therefore, if we obtain an approximation to $q$, we thereby obtain an approximation to $L$.

The domain of integration in Eq. (2.4) is represented as the shaded area in Figure 1. This figure shows that $E^{\prime \prime}=E^{\prime}$ in the domain of integration only at the point $E^{\prime \prime}=E^{\prime}=E$. This is the point at which the differential scattering cross section in Eq. (2.4) attains its maximum value.

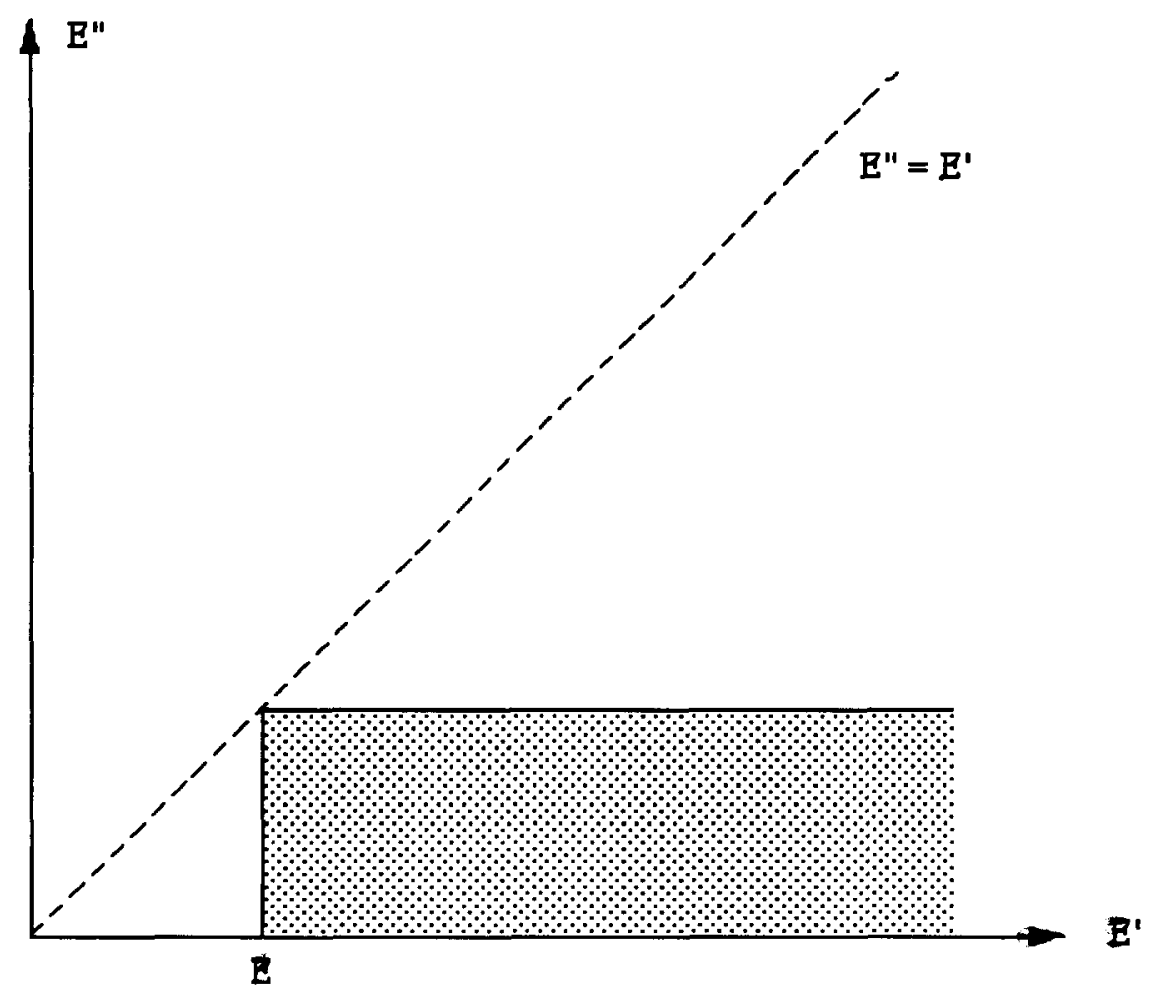

Fig. 1: Domain of Integration in Eq. (2.4). 
Now, using Eq. (2.1), we can write for $E^{\prime} \approx E$ and $E^{\prime \prime} \approx E$

$$
\begin{aligned}
& \phi\left(E^{\prime}\right)=\Phi\left(E^{\prime}\right)\left[\psi(E)+\left(E^{\prime}-E\right) \psi^{\prime}(E)+\frac{1}{2}\left(E^{\prime}-E\right)^{2} \psi^{\prime \prime}(E)+\cdots\right] \\
& \phi\left(E^{\prime \prime}\right)=\Phi\left(E^{\prime \prime}\right)\left[\psi(E)+\left(E^{\prime \prime}-E\right) \psi^{\prime}(E)+\frac{1}{2}\left(E^{\prime \prime}-E\right)^{2} \psi^{\prime \prime}(E)+\cdots\right]
\end{aligned}
$$

Introducing these expressions into Eq. (2.4), we obtain

$$
\begin{aligned}
q(E) & =\psi(E) F_{0}(E)+\psi^{\prime}(E) F_{1}(E)+\psi^{\prime \prime}(E) F_{2}(E)+\cdots \\
& =\left(\frac{\phi(E)}{\Phi(E)}\right) F_{0}(E)+\left(\frac{\phi(E)}{\Phi(E)}\right) F_{1}(E)+\left(\frac{\phi(E)}{\Phi(E)}\right)^{\prime \prime} F_{2}(E)+\cdots
\end{aligned}
$$

where

$$
\begin{aligned}
F_{n}(E) \equiv \frac{1}{n !} \int_{E^{\prime}=E}^{\infty} \int_{E^{\prime \prime}=0}^{E}[ & \Sigma_{S}\left(E^{\prime} \rightarrow E^{\prime \prime}\right) \Phi\left(E^{\prime}\right)\left(E^{\prime}-E\right)^{n} \\
& \left.-\Sigma_{S}\left(E^{\prime \prime} \rightarrow E^{\prime}\right) \boldsymbol{\Phi}\left(E^{\prime \prime}\right)\left(E^{\prime \prime}-E\right)^{n}\right] d E^{\prime \prime} d E^{\prime} .
\end{aligned}
$$

It should be noted that, from Eq. (1.19), the functions $F_{n}$ will decrease in magnitude with increasing $\mathrm{n}$. Therefore, because $\psi$ and its derivatives are $\mathrm{O}(1)$, the dots in Eq. (2.7) refer to terms that are strictly of order $F_{3}$.

It is given that $L$ satisfies Eq. (1.7). Thus, Eq. (2.5) implies that if $\phi(E)=M(E)$, then $q(E)=0$. The assumption that $\zeta(E)$ and $\psi(E)$ are bounded with bounded derivatives now means that we can repeat the above calculations [Eqs. (2.6) - (2.8)] with $\phi$ replaced by $M$, and the version of Eq. (2.7) that results is

$$
0=\left(\frac{M(E)}{\Phi(E)}\right) F_{0}(E)+\left(\frac{M(E)}{\Phi(E)}\right)^{\prime} F_{1}(E)+\left(\frac{M(E)}{\Phi(E)}\right)^{\prime \prime} F_{2}(E)+\cdots,
$$

where, as before, the dots refer to terms that are strictly of order $F_{3}$. Using this to eliminate $F_{0}(E)$ in Eq. (2.7), we obtain, after some routine algebraic manipulations,

$$
q(E)=F_{1}(E) \frac{M(E)}{\Phi(E)} \frac{d}{d E} \frac{\phi(E)}{M(E)}+F_{2}(E) \frac{\Phi(E)}{M(E)} \frac{d}{d E}\left(\frac{M(E)}{\Phi(E)}\right)^{2} \frac{d}{d E} \frac{\phi(E)}{M(E)}+\cdots
$$

Therefore, Eq. (2.5) can be written

$$
L \phi(E)=\frac{d}{d E} D_{A}(E) \frac{d}{d E} \frac{\phi(E)}{M(E)}+E_{\mathrm{A}} \quad,
$$

where 


$$
D_{A}(E)=F_{1}(E) \frac{M(E)}{\Phi(E)}
$$

and

$$
\mathcal{E}_{\mathrm{A}}=\frac{d}{d E} F_{2}(E) \frac{\Phi(E)}{M(E)} \frac{d}{d E}\left(\frac{M(E)}{\Phi(E)}\right)^{2} \frac{d}{d E} \frac{\phi(E)}{M(E)}+\cdots
$$

To obtain our generalized heavy gas model, we simply delete $\mathcal{E}_{\mathrm{A}}$ in Eq. (2.10). This amounts to deleting terms of order $F_{2}$ but retaining terms of order $F_{0}$ and $F_{1}$. Eq. (2.12) then gives the leading term in the resulting error for any choice of $\Phi$. large $E$ :

To estimate this error, let us consider a problem for which $\phi(E)$ has a $1 / E$ "tail" for

$$
\phi(E) \approx\left\{\begin{array}{ll}
M(E) & E \approx k T \\
\beta / E & E \gg>k T
\end{array},\right.
$$

where $\beta$ is a constant.

Now we consider two choices for $\Phi$. We first consider the "conventional" choice

$$
\Phi(E)=M(E) \quad,
$$

for which Eqs. (2.10) and (2.11) reduce to Corngold's first result, Eqs. (1.15) and (1.16). [However, for this problem, $\psi=\phi / \Phi=\phi / M$ becomes unbounded for large $E$, so we would argue that for large $E$, Eq. (2.14) is inappropriate.] Nevertheless, Eq. (2.12) reduces to

$$
\mathcal{E}_{\mathrm{A}}=\frac{d}{d E} F_{2}(E) \frac{d^{2}}{d E^{2}} \frac{\phi(E)}{M(E)}+\cdots
$$

Now, because of Eq. (2.13), we have

$$
\frac{\phi(E)}{M(E)} \approx(k T)^{2} \beta \frac{e^{E / k T}}{E^{2}} \quad E \gg>T,
$$

so Eq. (2.15) yields the estimate

$$
\mathcal{E}_{\mathrm{A}} \approx \beta \frac{d}{d E} F_{2}(E) \frac{e^{E / k T}}{E^{2}} \quad E \gg k T
$$

We next consider a choice of $\Phi$ which more accurately reflects the behavior of $\phi$ for large $E$. The $\Phi$ which makes our analysis simplest is $\Phi=\phi ; E q$. (2.12) then yields 


$$
\begin{aligned}
\mathcal{E}_{\mathrm{A}} & =\frac{d}{d E} F_{2}(E) \frac{\phi(E)}{M(E)} \frac{d}{d E}\left(\frac{M(E)}{\phi(E)}\right)^{2} \frac{d}{d E} \frac{\phi(E)}{M(E)}+\cdots \\
& =-\frac{d}{d E} F_{2}(E) \frac{\phi(E)}{M(E)} \frac{d^{2}}{d E^{2}} \frac{M(E)}{\phi(E)}+\cdots
\end{aligned}
$$

Using Eq. (2.16), this estimate becomes

$$
\mathcal{E}_{\mathrm{A}} \approx \frac{1}{k^{2} T^{2}} \frac{d}{d E} F_{2}(E) \quad E>>T T \quad,
$$

and it is clear that this is much smaller than the estimate (2.17).

It is neither necessary nor practical to use $\Phi=\phi$ in an actual application. However, any expression for $\Phi$ that behaves as $\phi$ for large $E$ will lead to an error estimate of the same order as Eq. (2.19).

In summary, our analysis has shown that the choice of $\Phi(E)$ can greatly affect the accuracy of the approximation of $L$ given by Eqs. (2.10) and (2.11) (with the term $\mathcal{E}_{\mathrm{A}}$ deleted). In particular, the conventional choice $F(E)=M(E)$ should not be optimal for problems containing a $1 / E$ "tail", and for $E \gg k T$.

\section{DERIVATION OF METHOD B}

Next, we describe a procedure for obtaining a result that has the same form as Eq. (2.10), but with different expressions for the diffusion coefficient $D(E)$ and the error $\mathcal{E}$. The starting point is now Eq. (1.1), into which we introduce the expressions (2.6). We directly obtain

$$
L \phi(E)=\psi(E) G_{0}^{\prime}(E)+\psi^{\prime}(E) G_{1}(E)+\psi^{\prime \prime}(E) G_{2}(E)+\cdots \quad,
$$

where $G_{0}(E)$ is uniquely defined by

$$
\begin{aligned}
& \frac{d}{d E} G_{0}(E)=L \Phi(E)=\int_{0}^{\infty}\left[\Sigma_{S}\left(E^{\prime} \rightarrow E\right) \Phi\left(E^{\prime}\right)-\Sigma_{S}\left(E \rightarrow E^{\prime}\right) \Phi(E)\right] d E^{\prime}, \\
& G_{0}(0)=G_{0}(\infty)=0,
\end{aligned}
$$

and $G_{n}(E)$ for $n \geq 1$ are defined by

$$
G_{n}(E)=\frac{1}{n !} \int_{0}^{\infty} \Sigma_{S}\left(E^{\prime} \rightarrow E\right) \Phi\left(E^{\prime}\right)\left(E^{\prime}-E\right)^{n} d E^{\prime}
$$

Then, from Eq. (1.19), the functions $G_{n}(\mathrm{E})$ decrease in magnitude with increasing $n$.

Now, we have seen in Sec. II that we can write

$$
L \phi(E)=\frac{d}{d E} q(E) \quad,
$$

so the right side of Eq. (3.1) must be of this form. Manipulating Eq. (3.1), we obtain 


$$
\begin{aligned}
L \phi(E)= & \frac{d}{d E}\left[\psi(E) G_{0}(E)+\psi^{\prime}(E)\left(G_{2}(E)-G_{3}^{\prime}(E)\right)+\psi^{\prime \prime}(E) G_{3}(E)\right] \\
& -\psi^{\prime}(E)\left[G_{0}(E)-G_{1}(E)+G_{2}^{\prime}(E)-G_{3}^{\prime}(E)\right]+\cdots
\end{aligned}
$$

Therefore, we must have

$$
G_{0}(E)=G_{1}(E)-G_{2}^{\prime}(E)+G_{3}^{\prime}(E)+\cdots,
$$

and using this to eliminate $G_{0}$ from Eq. (3.4), we obtain

$$
\begin{aligned}
L \phi(E)= & \frac{d}{d E}\left[\psi(E)\left(G_{1}(E)-G_{2}^{\prime}(E)+G_{3}^{\prime}(E)\right)+\psi^{\prime}(E)\left(G_{2}(E)-G_{3}^{\prime}(E)\right)\right. \\
& \left.+\psi^{\prime \prime}(E) G_{3}(E)+\cdots\right] \\
= & \frac{d}{d E}\left[\left(\frac{\phi(E)}{\Phi(E)}\right)\left(G_{1}(E)-G_{2}^{\prime}(E)+G_{3}^{\prime \prime}(E)\right)+\left(\frac{\phi(E)}{\Phi(E)}\right)^{\prime}\left(G_{2}(E)-G_{3}^{\prime}(E)\right)\right. \\
& \left.+\left(\frac{\phi(E)}{\Phi(E)}\right)^{\prime \prime} G_{3}(E)+\cdots\right] .
\end{aligned}
$$

We also know that $L M(E)=0$. Therefore, Eq. (3.5) gives

$$
0=\frac{M}{\Phi}\left(G_{1}-G_{2}^{\prime}+G_{3}^{\prime \prime}\right)+\left(\frac{M}{\Phi}\right)^{\prime}\left(G_{2}-G_{3}^{\prime \prime}\right)+\left(\frac{M}{\Phi}\right)^{\prime \prime} G_{3}+\cdots
$$

or

$$
G_{1}-G_{2}^{\prime}+G_{3}^{\prime \prime}=-\left(\frac{\Phi}{M}\right)\left(\frac{M}{\Phi}\right)^{\prime}\left(G_{2}-G_{3}^{\prime}\right)-\left(\frac{\Phi}{M}\right)\left(\frac{M}{\Phi}\right)^{\prime \prime} G_{3}+\cdots
$$

Introducing this into Eq. (3.5), we obtain, after some routine algebraic manipulation,

$$
L \phi(E)=\frac{d}{d E} D_{B}(E) \frac{d}{d E} \frac{\phi(E)}{M(E)}+\mathcal{E}_{\mathrm{B}},
$$

where

$$
D_{B}(E)=G_{2}(E) \frac{M(E)}{\Phi(E)}
$$

and 


$$
\begin{aligned}
\mathcal{E}_{\mathrm{B}}= & -\frac{d}{d E} G_{3}^{\prime}(E) \frac{M(E)}{\Phi(E)} \frac{d}{d E} \frac{\phi(E)}{M(E)} \\
& +\frac{d}{d E} G_{3}(E) \frac{\Phi(E)}{M(E)} \frac{d}{d E}\left(\frac{M(E)}{\Phi(E)}\right)^{2} \frac{d}{d E} \frac{\phi(E)}{M(E)}+\cdots
\end{aligned}
$$

We obtain a second generalized heavy gas model (model B) by deleting the term $\mathcal{E}_{\mathrm{B}}$ in Eq. (3.6). This amounts to deleting terms of order $G_{3}$, but retaining terms of order $G_{0}, G_{1}$, and $G_{2}$. The leading term in the resulting error is given by Eq. (3.8) for any choice of $\Phi$. If we choose $\Phi=M$, it is easily seen that Eqs. (3.6) and (3.7) reduce to Corngold's second result, Eqs. (1.16) and (1.17). In addition, for problems in which $\phi(E)$ is of the form (2.13), we obtain the error estimate

$$
\mathcal{E}_{\mathrm{B}} \approx k T \beta \frac{d}{d E} \frac{e^{E / k T}}{E^{2}}\left[-G_{3}^{\prime}(E)+\frac{G_{3}(E)}{k T}\right] \quad, \quad E \gg>T
$$

However, if we instead choose $\Phi(E)=\phi(E)$, we obtain

$$
\mathcal{E}_{\mathrm{B}} \approx-\frac{1}{k T} \frac{d}{d E}\left[G_{3}^{\prime}(E)+\frac{G_{3}(E)}{k T}\right] \quad, \quad E \gg k T,
$$

which is much smaller than the estimate (3.9).

Thus, as in Sec. II, we have a new generalized heavy gas model which, given a good choice of $\Phi$, should be substantially more accurate than the standard Heavy Gas Moded.

\section{COMPARISON OF METHODS A AND B}

The two approximations to $L$ that we have derived above differ due to the different definitions of the diffusion coefficients, $D_{A}(E)$ and $D_{B}(E)$. From Eqs. (2.11) and (3.7),

$$
D_{A}(E)=F_{1}(E) \frac{M(E)}{\Phi(E)}
$$

and

$$
D_{B}(E)=G_{2}(E) \frac{M(E)}{\Phi(E)}
$$

Therefore, the difference between $D_{A}$ and $D_{B}$ can be determined by estimating the difference between $F_{1}$ and $G_{2}$. Likewise, the relative accuracy of the two methods can be gauged by comparing $\mathcal{E}_{\mathrm{A}}$ and $\mathcal{E}_{\mathrm{B}}$.

By Eqs. (2.5) and (2.7),

$$
L \phi(E)=\frac{d}{d E}\left[\psi(E) F_{0}(E)+\psi^{\prime}(E) F_{1}(E)+\psi^{\prime \prime}(E) F_{2}(E)+\cdots\right],
$$

and by Eq. (3.5), 


$$
\begin{aligned}
L \phi(E)=\frac{d}{d E} & {\left[\psi(E)\left(G_{1}(E)-G_{2}^{\prime}(E)+G_{3}^{\prime \prime}(E)\right)\right.} \\
& \left.+\psi^{\prime}(E)\left(G_{2}(E)-G_{3}^{\prime}(E)\right)+\psi^{\prime \prime}(E) G_{3}(E)+\cdots\right] .
\end{aligned}
$$

These two equations imply

$$
\begin{aligned}
& F_{0}(E) \approx G_{1}(E)-G_{2}^{\prime}(E)+G_{3}^{\prime}(E), \\
& F_{1}(E) \approx G_{2}(E)-G_{3}^{\prime}(E), \\
& F_{2} \approx G_{3} .
\end{aligned}
$$

Combining the last of these three equations with Eqs. (2.12) and (3.8), we obtain

$$
\begin{aligned}
& \mathcal{E}_{\mathrm{A}} \approx \frac{d}{d E} G_{3}(E) \frac{\Phi(E)}{M(E)} \frac{d}{d E}\left(\frac{M(E)}{\Phi(E)}\right)^{2} \frac{d}{d E} \frac{\phi(E)}{M(E)} \\
& \mathcal{E}_{\mathrm{B}} \approx \frac{d}{d E} G_{3}(E) \frac{\Phi(E)}{M(E)} \frac{d}{d E}\left(\frac{M(E)}{\Phi(E)}\right)^{2} \frac{d}{d E} \frac{\phi(E)}{M(E)}-\frac{d}{d E} G_{3}^{\prime}(E) \frac{M(E)}{\Phi(E)} \frac{d}{d E} \frac{\phi(E)}{M(E)}
\end{aligned}
$$

Therefore, $\mathcal{E}_{\mathrm{A}}$ and $\mathcal{E}_{\mathrm{B}}$ are both of the order of $G_{3}$ and its derivatives. However, $\mathcal{E}_{\mathrm{A}}$ contains only a first derivative of $G_{3}$, while $\mathcal{E}_{\mathrm{B}}$ contains a second derivative. It therefore appears that in general, method $\mathrm{A}$ should be more accurate.

Also, Eqs. (4.1), (4.2), and (4.4) show that $D_{A}$ and $D_{B}$ differ by a term whose size is formally on the order of other terms that have been neglected. This term directly produces the second term on the right side of Eq. (4.7) (the term in $\mathcal{E}_{\mathrm{B}}$ causing the difference between $\mathcal{E}_{\mathrm{A}}$ and $\mathcal{E}_{\mathrm{B}}$ ). Therefore, the neglect of the formally small component of the diffusion coefficient in method $\mathrm{B}$ directly leads to the term in $\mathcal{E}_{\mathrm{B}}$ that appears to make method $\mathrm{B}$ less desirable than method $\mathrm{A}$.

\section{DISCUSSION}

We have derived two new Generalized Heavy Gas (or, Fokker-Planck) approximations to the infinite medium neutron spectrum equation, Eq. (1.1). Our analysis also provides error estimates indicating that both of the new methods should be more accurate than the standard Generalized Heavy Gas Model for $E \gg>T$, and that the first of the new methods derived here, Method A, should generally be more accurate than the second, method B. The only difference between Method A, Method B, and the standard Model, is in the definition of the diffusion coefficient $D(E)$.

Neither of the new methods has been tested numerically. However, we would like to suggest two procedures that may be of use in computer applications. Both procedures concern the definition of the function $\Phi(E)$, which is required well-approximate $\phi(E)$.

Let us write the heavy gas approximation to Eq. (1.1) in the form

$$
\Sigma_{A}(E) \phi(E)=R[\Phi] \phi(E)+Q(E) \quad,
$$


which explicitly shows that $R$ depends on $\Phi(E)$. Our first suggestion is that if a good estimate of $\phi$ is not readily available, then one could iterate on $\Phi$ in Eq. (5.1) by:

$$
\begin{aligned}
& \phi^{(0)}(E)=M(E) \\
& \Sigma_{A}(E) \phi^{(n+1)}(E)=R\left[\phi^{(n)}\right] \phi^{(n+1)}(E)+Q(E)
\end{aligned}
$$

The advantage of this procedure is that it could give more accurate results than the standard Generalized Heavy Gas Model. The disadvantages are that one has to re-evaluate the diffusion coefficient for every iteration, and that in the limit as $n \rightarrow \infty$, the solution of the original problem is not attained. [The errors $\mathcal{E}_{\mathrm{A}}$ and $\mathcal{E}_{\mathrm{B}}$ in Eqs. (4.6) and (4.7) do not vanish when $\Phi=\phi$.] Therefore, if one were to iterate in the above manner, it would probably be best to do so only a few times.

Our second suggestion is that for time-dependent problems, one choose $\Phi(E)$ for any given time step to be the value of $\phi(E)$ calculated from the previous time step. Then, provided the time steps are not too large, $\Phi(E)$ should be a good approximation to $\phi(E)$. The advantage of this procedure is that it should produce a satisfactory choice of $\Phi(E)$ without having to iterate; the disadvantage is that one has to re-evaluate the diffusion coefficient for every time step.

We have already mentioned that the method in this paper can be applied to the derivation of Fokker-Planck approximations for other physical processes, such as Compton scattering (Pomraning, 1973, Cooper, 1971, Larsen et. al., 1985), and that the resulting numerical algorithms should produce solutions which more accurately represent the solution of the original problem. Both these subjects are, however, beyond the scope of the present paper.

\section{ACKNOWLEDGEMENTS}

The work by the first author (E.W.L.) was supported by the National Science Foundation under Grant No. EET-8721680.

\section{REFERENCES}

Cooper G. (1971) Phys. Rev. D 3, 2312.

Corngold N. (1960) Ann. Phys. (NY) 11, 338.

Corngold N. (1962) Proc. Brookhaven National Laboratory Conference on Neutron Thermalization, Brookhaven National Laboratory Report BNL-719, 1075.

Hurwitz H., Nelkin M.S., and Habetler E.J. (1956) Nucl. Sci. Eng. 1, 280.

Larsen E.W., Levermore C.D., Pomraning G.C., and Sanderson J.G. (1985) J. Comp. Phys. 61, 359.

Oppenheim, I., Schuler, K.E., and Weiss, G.H. (1977) Stochastic Processis in Chemical Physics: The Master Equation, MIT Press, Cambridge, Mass.

Pomraning, G.C. (1973) The Equations of Radiation Hydrodynamics, Pergamon Press, Oxford.

Pitcher, H.H.W. (1963) Report AEEW-M350.

Sachs R.G. and Teller E. (1941) Phys. Rev. 60, 18.

Schaefer G. and Allsop K. (1962) Proc. Brookhaven National Laboratory Conf. on Neutron Thermalization, Brookhaven National Laboratory Report BNL-719, 614.

Wilkins J.E., Jr. (1944) AEC Report CP-2481. Also in (1948) Annals of Mathematics 49, 189. 